\title{
Time-Varying Modification of Reference Model for Adaptive Control with Performance Optimization
}

\author{
Nhan T. Nguyen \\ NASA Ames Research Center, Moffett Field, CA 94035 \\ Kelley E. Hashemi \\ Universities Space Research Association, Moffett Field, CA 94035
}

\begin{abstract}
This paper presents a new adaptive control approach that involves a performance optimization objective. The control synthesis involves the design of a performance optimizing adaptive controller from a subset of control inputs. The resulting effect of the performance optimizing adaptive controller is to modify the initial reference model into a time-varying reference model which satisfies the performance optimization requirement obtained from an optimal control problem. The time-varying reference model modification is accomplished by the real-time solutions of the time-varying Riccati and Sylvester equations coupled with the least-squares parameter estimation of the sensitivities of the performance metric. The effectiveness of the proposed method is demonstrated by an application of maneuver load alleviation control for a flexible aircraft.
\end{abstract}

\section{INTRODUCTION}

In this work, we develop a multi-objective performance-based model-reference adaptive control (MRAC) with the goal of providing adaptation while seeking to optimize a performance metric for an uncertain plant. The plant is assumed to have sufficient control redundancy to achieve the objective of performance optimization in addition to the usual MRAC objective of maintaining tracking performance in the presence of matched uncertainty. The performance metric is available from sensor measurements but its sensitivities with respect to the state and control variables are assumed to be unknown. Least-squares parameter estimation is designed to estimate the performance sensitivities. The performance optimization is cast as a multi-objective gradient optimization problem. The gradient of the Hamiltonian function is computed in real-time from the estimates of the performance sensitivities. This results in time-varying modified Riccati and Sylvester equations which produce time-varying control gains. The performance optimizing

\footnotetext{
Technical Group Lead and Senior Research Scientist, Intelligent Systems Division, AIAA Associate Fellow, nhan.t.nguyen@nasa.gov

NASA Post-Doctoral Fellow, Intelligent Systems Division, kelley.e.hashemi@nasa.gov
}

controller is then augmented to the reference model. This results in a time-varying modified reference model in order for MRAC to achieve simultaneously the original tracking performance objective as well as the performance optimization objective.

Reference model modification has been investigated extensively but time-varying modification is generally not considered. One approach is to include a tracking error feedback term in the reference model to improve transient performance [1]. The pseudo-control hedging is another method which modifies the reference command signal in the presence of control saturation [2]. Multiobjective adaptive control has also been developed [3].

In the proposed approach, a number of new contributions are made. The gradient optimization leads to a set of modified time-varying Riccati and Sylvester equations whereby the standard weighting matrices $Q$ and $R$ are modified to incorporate the estimates of the performance sensitivities. The reference command signal is designed to be generated from a reference command generating function in order to allow the performance optimizing controller to be obtained explicitly from the gradient optimization in a feedback form. The resultant timevarying modification of the reference model requires the standard MRAC to use a time-varying weighting matrix computed from the modified Riccati equation. A simulation of maneuver load alleviation control demonstrates the effectiveness of the proposed method.

\section{Performance Optimizing Adaptive Control}

Consider a plant model

$$
\dot{x}=A x+B\left[u+\Theta^{* \top} \Phi(x)\right]
$$

subject to $x\left(t_{0}\right)=x_{0}$, where $x \in \mathbb{R}^{n}$ is the state vector, $u \in \mathbb{R}^{m}$ is the control vector, $\Theta^{*} \in \mathbb{R}^{l \times m}$ is an unknown constant matrix, $\Phi(x) \in \mathbb{R}^{l}$ is a known regressor function, and $A \in \mathbb{R}^{n \times n}$ and $B \in \mathbb{R}^{n \times m}$ are known matrices. The plant states are fully accessible. The pair $(A, B)$ is 
assumed to be controllable. The plant is associated with a performance metric

$$
y=C x+D u
$$

where $y \in \mathbb{R}^{k}, k<n$, is a vector of performance metrics which are available from measurements, and $C \in \mathbb{R}^{k \times n}$ and $D \in \mathbb{R}^{k \times m}$ are sensitivity matrices which are assumed to be unknown. The goal is to design an adaptive controller that tracks a reference model as well as optimizes the performance metrics. To that end, we introduce a reference command generating function

$$
\begin{gathered}
\dot{z}=A_{r} z+z_{r} \\
r=C_{r} z
\end{gathered}
$$

subject to $z\left(t_{0}\right)=z_{0}$, where $z \in \mathbb{R}^{p}, p \geq q, z_{r} \in \mathbb{R}^{p}$ is a constant command signal, $r \in \mathbb{R}^{q}$ is a reference command signal with $q \leq m, A_{r} \in \mathbb{R}^{p \times p}$ is a stable matrix, and $C_{r} \in \mathbb{R}^{q \times p}$. Consider the following adaptive controller:

$$
u=u_{n o m}+u_{a d}+u_{p}
$$

where $u_{\text {nom }}=K_{x} x+K_{r} r, u_{a d}=-\Theta^{\top} \Phi(x)$, and $u_{p}$ is a performance optimizing controller to be determined.

The incremental performance metric due to the performance optimizing controller $u_{p}$ is defined as

$$
\Delta y=C x+D u_{p}
$$

Let $\Delta \hat{y}$ be the estimate of $\Delta y$ where

$$
\Delta \hat{y}=\hat{C} x+\hat{D} u_{p}
$$

with $\hat{C}$ and $\hat{D}$ being the estimates of $C$ and $D$, respectively. Next, the performance metric estimation error is computed as

$$
e_{y}=\Delta \hat{y}-\Delta y=\tilde{C} x+\tilde{D} u_{p}
$$

where $\tilde{C}=\hat{C}-C$ and $\tilde{D}=\hat{D}-D$ are the estimation errors of $C$ and $D$, respectively. $\hat{C}$ and $\hat{D}$ can be estimated from a least-squares gradient method that minimizes the following cost function

$$
J=\frac{1}{2} e_{y}^{\top} e_{y}
$$

This results in the least-squares gradient adaptive laws

$$
\begin{gathered}
\dot{\hat{C}}^{\top}=-\Gamma_{C} \frac{\partial J}{\partial \tilde{C}}=-\Gamma_{C} x e_{y}^{\top} \\
\dot{\hat{D}}^{\top}=-\Gamma_{D} \frac{\partial J}{\partial \tilde{D}}=-\Gamma_{D} u_{p} e_{y}^{\top}
\end{gathered}
$$

where $\Gamma_{C}=\Gamma_{C}^{\top} \in \mathbb{R}^{n \times n}>0$ and $\Gamma_{D}=\Gamma_{D}^{\top} \in \mathbb{R}^{m \times m}>0$ are adaptation rate matrices. To design the performance optimizing controller, consider the ideal plant with $u_{a d}^{*}=$ $-\Theta^{* \top} \Phi(x)$

$$
\dot{x}=A_{m} x+B_{m} r+B u_{p}
$$

where $A_{m}=A+B K_{x}$ and is Hurwitz, and $B_{m}=B K_{r}$.
This problem is cast as a multi-objective optimization of the ideal plant using the following infinite-timehorizon cost function:

$$
J=\frac{1}{2} \int_{t_{0}}^{t_{f} \rightarrow \infty}\left(\Delta \hat{y}^{\top} Q \Delta \hat{y}+u_{p}^{\top} R u_{p}\right) d t
$$

where $Q=Q^{\top}>0 \in \mathbb{R}^{k \times k}$ and $R=R^{\top}>0 \in \mathbb{R}^{m \times m}$ subject to the plant dynamics in Eq. (12). The Hamiltonian function is defined as

$$
H=\frac{1}{2} \Delta \hat{y}^{\top} Q \Delta \hat{y}+\frac{1}{2} u_{p}^{\top} R u_{p}+\mu^{\top}\left(A_{m} x+B_{m} r+B u_{p}\right)
$$

where $\mu \in \mathbb{R}^{n}$ is an adjoint vector. Then, the necessary conditions are obtained as

$$
\dot{\mu}=-\frac{\partial H^{\top}}{\partial x}=-\hat{C}^{\top} Q\left(\hat{C} x+\hat{D} u_{p}\right)-A_{m}^{\top} \mu
$$

subject to the transversality condition $\mu\left(t_{f}\right)=0$, and

$$
\frac{\partial H^{\top}}{\partial u_{p}}=\hat{D}^{\top} Q\left(\hat{C} x+\hat{D} u_{p}\right)+R u_{p}+B^{\top} \mu=0
$$

The optimal control $u_{p}$ is then obtained as

$$
u_{p}=-\left(R+\hat{D}^{\top} Q \hat{D}\right)^{-1}\left(B^{\top} \mu+\hat{D}^{\top} Q \hat{C} x\right)
$$

Using the assumed solution $\mu=W x+V z+U z_{r}$, the following equations are obtained:

$$
\begin{gathered}
\dot{W}+W \bar{A}+\bar{A}^{\top} W-W B \bar{R}^{-1} B^{\top} W+\bar{Q}=0 \\
\dot{V}+\left(\bar{A}^{\top}-W B \bar{R}^{-1} B^{\top}\right) V+V A_{r}+W B_{m} C_{r}=0 \\
\dot{U}+\left(\bar{A}^{\top}-W B \bar{R}^{-1} B^{\top}\right) U+V=0
\end{gathered}
$$

subject to the transversality conditions $W\left(t_{f}\right)=0$, $V\left(t_{f}\right)=0$, and $U\left(t_{f}\right)=0$, where

$$
\begin{gathered}
\bar{A}=A_{m}-B \bar{R}^{-1} \hat{D}^{\top} Q \hat{C} \\
\bar{Q}=\hat{C}^{\top} Q\left(I-\hat{D} \bar{R}^{-1} \hat{D}^{\top} Q\right) \hat{C} \\
\bar{R}=R+\hat{D}^{\top} Q \hat{D}
\end{gathered}
$$

$Q$ is chosen such that $\bar{Q}>0$ which implies $\hat{D} \bar{R}^{-1} \hat{D}^{\top} Q<I$. Equation (18) is a time-varying differential Riccati equation with the time-varying matrices $\bar{A}$, $\bar{Q}$, and $\bar{R}$ which are updated at each time step as $\hat{C}$ and $\hat{D}$ are computed from the least-squares gradient adaptive laws. The existence of the solution of a time-varying differential Riccati equation depends on the properties of the time-varying matrices $\bar{A}, \bar{Q}$, and $\bar{R}$.

Theorem 1: Let $\bar{A}=\bar{A}^{*}+\delta_{\bar{A}}, \bar{Q}=\bar{Q}^{*}+\delta_{\bar{Q}}>0$, and $\bar{R}=\bar{R}^{*}+\delta_{\bar{R}}>0$ where $\bar{A}^{*}, \bar{Q}^{*}$, and $\bar{R}^{*}$ are some constant matrices. If $\delta_{\bar{A}}, \delta_{\bar{Q}}$, and $\delta_{\bar{R}}$ are at least piecewise continuous for all $t \in[0, \infty) ; \lim _{t \rightarrow \infty} \delta_{\bar{A}}=0, \lim _{t \rightarrow \infty} \delta_{\bar{Q}}=0$, and $\lim _{t \rightarrow \infty} \delta_{\bar{R}}=0$; and furthermore, $\dot{\delta}_{\bar{A}}, \dot{\delta}_{\bar{Q}}$, and $\dot{\delta}_{\bar{R}}$ are uniformly continuous; then $\bar{A}, \bar{Q}$, and $\bar{R}$ tend to 
their constant solutions $\bar{A}^{*}, \bar{Q}^{*}$, and $\bar{R}^{*}$, respectively, as $t \rightarrow \infty$. Consequently, the solution of the time-varying differential Riccati equation exists and also tends to its constant solution in the limit as $t_{f} \rightarrow \infty$.

Proof: The Hamiltonian system for a regulator design with $V=U=0$ is defined as

$$
\left[\begin{array}{c}
\dot{x} \\
\dot{\mu}
\end{array}\right]=\left[\begin{array}{cc}
\bar{A} & -B \bar{R}^{-1} B \\
-\bar{Q} & -\bar{A}^{\top}
\end{array}\right]\left[\begin{array}{l}
x \\
\mu
\end{array}\right]=H\left[\begin{array}{l}
x \\
\mu
\end{array}\right]
$$

where $H$ is the time-varying Hamiltonian matrix. The solution of Eq. (18) depends continuously on the timevarying stable eigenvalues of $H$ in frozen time. For a first-order SISO system, it can be shown that $\|\lambda(H)\|^{2} \leq$ $\left\|\bar{A}^{*}+\delta_{\bar{A}}\right\|^{2}+\|B\|^{2}\left\|\left(\bar{R}^{*}+\delta_{\bar{R}}\right)^{-1}\right\|\left\|\bar{Q}^{*}+\delta_{Q}\right\|$. Therefore, $\delta_{\bar{A}}, \delta_{\bar{Q}}$, and $\delta_{\bar{R}}$ must be at least piecewise continuous and uniformly bounded for all $t \in[0, \infty)$. Let $\Lambda=$ $\operatorname{diag}\left(-\Lambda_{H}, \Lambda_{H}\right)$ be the eigenvalue matrix where $\Lambda_{H}$ is the diagonal matrix of positive real eigenvalues of $H$. Then, the transition matrix $\Phi\left(\tau, \tau_{0}\right)$ where $\tau=t_{f}-t$ is the time-to-go variable is defined as

$$
\frac{d \Phi}{d \tau}=-\Lambda_{H} \Phi
$$

such that $\Phi\left(\tau_{0}, \tau_{0}\right)=I$. Then, $\Phi\left(\tau, \tau_{0}\right)$ is exponentially stable if there exists $\varepsilon>0$ such that $\left\|\dot{\Lambda}_{H}\right\| \leq \varepsilon$. This implies that $\dot{\delta}_{\bar{A}}, \dot{\delta}_{\bar{Q}}$, and $\dot{\delta}_{\bar{R}}$ are bounded for all $t \in[0, \infty)$. Moreover, we require $\bar{A} \rightarrow \bar{A}^{*}, \bar{R} \rightarrow \bar{R}^{*}$, and $\bar{Q} \rightarrow \bar{Q}^{*}$ uniformly as $t \rightarrow \infty$. This implies $\lim _{t \rightarrow \infty} \delta_{\bar{A}}=0$, $\lim _{t \rightarrow \infty} \delta_{\bar{Q}}=0$, and $\lim _{t \rightarrow \infty} \delta_{\bar{R}}=0$. Furthermore, $\overline{\bar{A}} \rightarrow 0$, $\dot{\bar{R}} \rightarrow 0, \dot{\bar{Q}} \rightarrow 0$ as $t \rightarrow \infty$ which require $\lim _{t \rightarrow \infty} \dot{\delta}_{\bar{A}}=0$, $\lim _{t \rightarrow \infty} \dot{\delta}_{\bar{Q}}=0$, and $\lim _{t \rightarrow \infty} \dot{\delta}_{\bar{R}}=0$. Then, $\dot{\delta}_{\bar{A}}, \dot{\delta}_{\bar{Q}}$, and $\dot{\delta}_{\bar{R}}$ are uniformly continuous according to the Barbalat's lemma. The solution of $W$ as $t \rightarrow \infty$ is given by [4]

$$
W=\left(X_{21}+X_{22} S\right)\left(X_{11}+X_{12} S\right)^{-1}
$$

where

$$
S=\Phi\left(\tau, \tau_{0}\right) S\left(t_{f}\right) \Phi\left(\tau, \tau_{0}\right)
$$

with $X_{i j}, i, j=1,2$ as the right eigenvector partitioned matrix such that $X \Lambda X^{-1}=H$, and $S\left(t_{f}\right)$ computed from Eq. (26). Let $W=W^{*}+\delta_{W}$ be the solution of the timevarying Riccati equation. Then, substituting into Eq. (18) and taking the limit as $t \rightarrow \infty$ yield

$$
\begin{gathered}
W^{*} \bar{A}^{*}+\bar{A}^{* \top} W^{*}-W^{*} B \bar{R}^{*-1} B^{\top} W^{*}+\bar{Q}^{*}=0 \\
\dot{\delta}_{W}+\delta_{W} \bar{A}^{*}+\bar{A}^{* \top} \delta_{W}-\delta_{W} B \bar{R}^{*-1} B^{\top} \delta_{W} \\
\quad-\delta_{W} B \bar{R}^{*-1} B^{\top} W^{*}-W^{*} B \bar{R}^{*-1} B^{\top} \delta_{W}=0
\end{gathered}
$$

Now, transforming Eq. (29) into the time-to-go variable gives

$$
\begin{aligned}
-\frac{d \delta_{W}}{d \tau} & +\delta_{W} \bar{A}^{*}+\bar{A}^{* \top} \delta_{W}-\delta_{W} B \bar{R}^{*-1} B^{\top} \delta_{W} \\
& -\delta_{W} B \bar{R}^{*-1} B^{\top} W^{*}-W^{*} B \bar{R}^{*-1} B^{\top} \delta_{W}=0
\end{aligned}
$$

subject to $\delta_{W}(\tau=0)=0$ since $W\left(t_{f}\right)=0$. The solution of Eq. (29) yields $\delta_{W}=0$ and $\dot{\delta}_{W}=0$ as $t_{f} \rightarrow \infty$. Thus, $W \rightarrow W^{*}$ as $t_{f} \rightarrow \infty$ which implies $\dot{W} \rightarrow 0$.

Equation (19) is a time-varying differential Sylvester equation. The existence of the solution of Eq. (19) can be established from Theorem 1 . If $W \rightarrow \bar{W}^{*}$, then the matrix $\bar{A}-B \bar{R}^{-1} B^{\top} W$ tends to a constant Hurwitz matrix $A_{c}=$ $\bar{A}^{*}-B \bar{R}^{*-1} B^{\top} W^{*}$. Equation (19) is transformed into the time-to-go variable as

$$
\frac{d V}{d \tau}=A_{c}^{\top} V+V A_{r}+W B_{m} C_{r}
$$

subject to $V(\tau=0)=0$. Since $A_{r}$ is a stable matrix, and $A_{r}$ can be chosen such that $\lambda_{i}\left(A_{c}\right)+\lambda_{j}\left(A_{r}\right) \neq 0$ for all $i$ and $j$, then it follows that $V$ is bounded as $t_{f} \rightarrow \infty$ and tends to a constant solution of the following algebraic Sylvester equation:

$$
A_{c}^{\top} V^{*}+V^{*} A_{r}+W^{*} B_{m} C_{r}=0
$$

Since $V^{*}$ exists, $U^{*}$ is bounded as $t_{f} \rightarrow \infty$ and tends to the following solution:

$$
U^{*}=-A_{c}^{-\top} V^{*}
$$

Thus, the solutions of Eqs. (18), (19), and (20) are computed from their corresponding time-varying algebraic equations. The performance optimizing controller $u_{p}$ is then expressed as

$$
u_{p}=\bar{K}_{x} x+\bar{K}_{z} z+\bar{K}_{z_{r}} z_{r}
$$

where $\bar{K}_{x}=-\bar{R}^{-1}\left(B^{\top} W+\hat{D}^{\top} Q \hat{C}\right), \bar{K}_{z}=-\bar{R}^{-1} B^{\top} V$, and $\bar{K}_{z_{r}}=\bar{R}^{-1} B^{\top} A_{c}^{-\top} V$.

The optimal control solution can also be formulated by using an alternative expression of the adjoint solution $\mu=W x+T$ where $T=V z+U z_{r}$ is the assumed solution. This results in the the following differential equation:

$$
\dot{T}+\left(\bar{A}^{\top}-W B_{p} \bar{R}^{-1} B_{p}^{\top}\right) T+W B_{m} r=0
$$

subject to $T\left(t_{f}\right)=0$ in lieu of Eqs. (19) and (20). Since $W(t) \rightarrow \bar{W}^{*}$ and $A_{c}$ is Hurwitz, it follows that $T$ is bounded as $t_{f} \rightarrow \infty$ and tends to the following solution:

$$
T^{*}=-A_{c}^{-\top} W^{*} B_{m} r-\sum_{n=1}^{\infty}(-1)^{n}\left(A_{c}^{\top}\right)^{-n-1} W^{*} B_{m} r^{(n)}
$$

Note that the series solution of $T^{*}$ is not a closed-form solution. This illustrates the advantage of the use of the reference command generating function.

Note that the uniform continuity condition for $\dot{\delta}_{\bar{A}}, \dot{\delta}_{\bar{Q}}$, and $\dot{\delta}_{\bar{R}}$ requires the parameter convergence of $\hat{C}$ and $\hat{D}$ to some constant matrices $\bar{C}$ and $\bar{D}$, respectively. 
Theorem 2: The least-squares gradient adaptive laws ensures a parameter convergence of $\hat{C}(t)$ and $\hat{D}(t)$ to some constant solutions $\bar{C}$ and $\bar{D}$, respectively.

Proof: Choose a Lyapunov candidate function

$$
V=\operatorname{trace}\left(\tilde{C} \Gamma_{C}^{-1} \tilde{C}^{\top}+\tilde{D} \Gamma_{D}^{-1} \tilde{D}^{\top}\right)
$$

for the ideal plant in Eq. (12). Then, $\dot{V}$ is evaluated as

$$
\begin{aligned}
& \dot{V}(\tilde{C}, \tilde{D})=-2 e_{y}^{\top} \tilde{C} x-2 e_{y}^{\top} \tilde{D} u_{p} \\
& \quad=-2\left(\tilde{C} x+\tilde{D} u_{p}\right)^{\top}\left(\tilde{C} x+\tilde{D} u_{p}\right)=-2 e_{y}^{\top} e_{y} \leq 0
\end{aligned}
$$

Thus, $\tilde{C} \in \mathscr{L}_{2} \cap \mathscr{L}_{\infty}$ and $\tilde{D} \in \mathscr{L}_{2} \cap \mathscr{L}_{\infty}$, but $x \in \mathscr{L}_{\infty}$ and $u_{p} \in \mathscr{L}_{\infty}$. It follow that $\bar{K}_{x} \in \mathscr{L}_{\infty}, \bar{K}_{z} \in \mathscr{L}_{\infty}$, and $\bar{K}_{z r} \in \mathscr{L}_{\infty}$. Therefore, for the ideal plant, $\dot{x} \in \mathscr{L}_{\infty}$. We note that $\ddot{V}$ is bounded since $\dot{x} \in \mathscr{L}_{\infty}$ and $\dot{u}_{p} \in \mathscr{L}_{\infty}$ by the virtue of $\dot{z} \in$ $\mathscr{L}_{\infty}, \dot{\bar{K}}_{x} \in \mathscr{L}_{\infty}, \dot{\bar{K}}_{z} \in \mathscr{L}_{\infty}$, and $\dot{\bar{K}}_{z r}(t) \in \mathscr{L}_{\infty}$. Therefore, $\dot{V}$ is uniformly continuous. Invoking the Barbalat's lemma, $\dot{V} \rightarrow 0$ as $t \rightarrow \infty$. This implies $e_{y} \rightarrow 0$ as $t \rightarrow \infty$. Since $\ddot{\hat{C}}$ an $\ddot{\hat{D}}$ are bounded, it follows that $\dot{\hat{C}}$ and $\dot{\hat{D}}$ are also uniformly continuous with $\dot{\hat{C}} \rightarrow 0$ and $\dot{\hat{D}} \rightarrow 0$ as $t \rightarrow \infty$. Let

$$
\begin{gathered}
\Delta \hat{C}=\hat{C}(t)-\hat{C}(t-\Delta t)=-\int_{t-\Delta t}^{t} \Gamma_{c} x e_{y}^{\top} d \tau \\
\Delta \hat{D}=\hat{D}(t)-\hat{D}(t-\Delta t)=-\int_{t-\Delta t}^{t} \Gamma_{D} u_{p} e_{y}^{\top} d \tau
\end{gathered}
$$

Then, $\Delta \hat{C} \rightarrow 0$ and $\Delta \hat{D} \rightarrow 0$ since $e_{y} \rightarrow 0$ as $t \rightarrow \infty$. This implies that $\hat{C} \rightarrow \bar{C}$ and $\hat{D} \rightarrow \bar{D}$ as $t \rightarrow \infty$. Therefore, $\bar{A}, \bar{Q}$, $\bar{R}, \bar{K}_{x}, \bar{K}_{z}$, and $\bar{K}_{z r}$ all converge to the constant matrices $\bar{A}^{*}, \bar{Q}^{*}, \bar{R}^{*}, \bar{K}_{x}^{*}, \bar{K}_{z}^{*}$, and $\bar{K}_{z r}^{*}$, respectively. Note that $\hat{C}$ and $\hat{D}$ do not necessarily converge to the true unknown matrices $C$ and $D$, respectively. This would require the persistent excitation condition which cannot be verified for a closed-loop system.

The closed-loop plant with the performance optimizing controller $u_{p}$ now becomes

$$
\begin{aligned}
\dot{x}=\left(A_{m}+B \bar{K}_{x}\right) x+B_{m} r+ & B\left(K_{z} z+K_{z r} z_{r}\right) \\
& +B\left[u_{a d}+\Theta^{* \top} \Phi(x)\right]
\end{aligned}
$$

Then, the performance optimizing reference model is expressed as

$$
\dot{x}_{m}^{*}=A_{m}^{*} x_{m}^{*}+B_{m} r+B K_{z}^{*} z+B K_{z_{r}}^{*} z_{r}
$$

where $A_{m}^{*}=A_{m}+B \bar{K}_{x}^{*}$. Let $\hat{x}_{m}$ be the estimate of $x_{m}^{*}$. Then, the time-varying performance optimizing reference model is given by

$$
\dot{\hat{x}}_{m}=\left(A_{m}+B \bar{K}_{x}\right) \hat{x}_{m}+B_{m} r+B K_{z} z+B K_{z_{r}} z_{r}
$$

Thus, $\hat{x}_{m} \rightarrow x_{m}^{*}$ as $t \rightarrow \infty$. Let $e=\hat{x}_{m}-x$ be the tracking error based on the time-varying modified reference model. Then, the tracking error equation becomes

$$
\dot{e}=\left(A_{m}+B \bar{K}_{x}\right) e+B \tilde{\Theta}^{\top} \Phi(x)
$$

The new MRAC update law is given by

$$
\dot{\Theta}=-\Gamma \Phi(x) e^{\top} W B
$$

where $W$ is the time-varying weighting matrix computed from the Riccati equation which replaces the constant weighting matrix $P$ computed from the Lyapunov equation in the standard MRAC update law. Robust modification can be used with the new MRAC update law such as the optimal control modification [5] given by

$$
\dot{\Theta}=-\Gamma \Phi(x)\left[e^{\top} W-v \Phi^{\top}(x) \Theta B^{\top} W\left(A_{m}+B \bar{K}_{x}\right)^{-1}\right] B
$$

where $v>0$ is a modification parameter.

The time-varying weighting matrix $W$ is obtained from the time-varying algebraic Riccati equation

$$
W \bar{A}+\bar{A}^{\top} W-W B \bar{R}^{-1} B^{\top} W+\bar{Q}=0
$$

Equivalently, the time-varying algebraic Riccati equation can be approximated by a stable time-varying differential Riccati equation integrated forward in time as follows:

$$
\varepsilon \dot{W}=W \bar{A}+\bar{A}^{\top} W-W B \bar{R}^{-1} B^{\top} W+\bar{Q}
$$

with $W(0)=W_{0}>0$ and $0<\varepsilon<1$ is a small positive constant. Using the singular perturbation argument, we transform Eq. (48) into the stretch time variable $t^{*}=\frac{t}{\varepsilon}$ :

$$
\frac{d W}{d t}=W \bar{A}\left(t^{*}\right)+\bar{A}\left(t^{*}\right)^{\top} W-W B \bar{R}\left(t^{*}\right)^{-1} B^{\top} W+\bar{Q}\left(t^{*}\right)
$$

with $W\left(t_{0}^{*}\right)=W_{0}$. By letting $\varepsilon \rightarrow 0$ in Eq. (48) which corresponds to $t^{*}=\mathscr{O}\left(\frac{1}{\varepsilon}\right) \rightarrow \infty$, the asymptotic outer solution of Eq. (48) is then obtained from Eq. (47). The inner solution of Eq. (48) corresponding to $t=\mathscr{O}(\varepsilon)$ obtained from Eq. (49) gives

$$
\frac{d W_{i}}{d t^{*}} \approx W_{0} \bar{A}_{0}+\bar{A}_{0}^{\top} W_{0}-W_{0} B \bar{R}_{0}^{-1} B^{\top} W_{0}+\bar{Q}_{0}=\sigma
$$

where $\sigma$ is a small-value matrix. The subscript $i$ denotes the inner solution. The subscript 0 denotes the value of a matrix evaluated at $t^{*}=t_{0}^{*}$ which depends on the initial conditions $\hat{C}\left(t_{0}\right)=C_{0}$ and $\hat{D}\left(t_{0}\right)=D_{0}$. It follows that $\left\|\dot{W}_{i}\right\|=\frac{\sigma}{\varepsilon}$. Equation (48) can be expressed in a Hamiltonian system as

$$
\varepsilon\left[\begin{array}{l}
\dot{p} \\
\dot{\lambda}
\end{array}\right]=\left[\begin{array}{cc}
-\bar{A} & B \bar{R}^{-1} B^{\top} \\
\bar{Q} & \bar{A}^{\top}
\end{array}\right]\left[\begin{array}{l}
p \\
\lambda
\end{array}\right]=-H\left[\begin{array}{l}
p \\
\lambda
\end{array}\right]
$$

where $\lambda=W p$ with $p\left(t_{0}\right)=p_{0}$ and $\lambda\left(t_{0}\right)=W_{0} p_{0}$. The transition matrix $\Phi\left(t, t_{0}\right)$ computed by

$$
\frac{d \Phi}{d t}=-\frac{1}{\varepsilon} \Lambda_{H} \Phi
$$


is exponentially stable. The solution is then given by Eq. (26) with $S=\Phi\left(t, t_{0}\right) S(0) \Theta\left(t, t_{0}\right)$ [4]. As $\varepsilon \rightarrow 0$ in the limit for the asymptotic outer solution, $S \rightarrow 0$ and $W \rightarrow W_{21} W_{11}^{-1}$ which is the solution of the time-varying algebraic Riccati equation. Moreover, the outer solution also yields $\dot{W}=0$ as $t \rightarrow \infty$ corresponding to the constant solution $W^{*}$ when $\hat{C} \rightarrow \bar{C}$ and $\hat{D} \rightarrow \bar{D}$.

The stability of the optimal control modification adaptive law can be shown in the following proof:

Proof: Choose a Lyapunov candidate function

$$
V=e^{\top} W e+\operatorname{trace}\left(\tilde{\Theta}^{\top} \Gamma^{-1} \tilde{\Theta}\right)
$$

Then, $\dot{V}(e, \tilde{\Theta})$ is evaluated as

$$
\begin{aligned}
\dot{V}= & -e^{\top}\left[\dot{W}+W\left(A_{m}+B \bar{K}_{x}\right)+\left(A_{m}+B \bar{K}_{x}\right)^{\top} W\right] e \\
& +2 v \Phi^{\top}(x) \Theta B^{\top} W\left(A_{m}+B \bar{K}_{x}\right)^{-1} B \tilde{\Theta}^{\top} \Phi(x)
\end{aligned}
$$

Using the outer solution of the singularly perturbed differential Riccati equation (48), we get

$$
\begin{gathered}
\dot{V}=-e^{\top} \mathscr{Q} e+2 v \Phi^{\top}(x) \Theta B^{\top} W\left(A_{m}+B \bar{K}_{x}\right)^{-1} B \tilde{\Theta}^{\top} \Phi(x) \\
\leq-c_{1}\|e\|^{2}-v c_{2}\|\Phi(x)\|^{2}\left(\|\tilde{\Theta}\|-c_{3}\right)^{2}+v c_{2} c_{3}^{2}\|\Phi(x)\|^{2}
\end{gathered}
$$

where $\mathscr{Q}=\bar{Q}+W B \bar{R}^{-1} B^{\top} W, c_{1}=\inf _{t} \lambda_{\min }(\mathscr{Q})>0$, $c_{2}=\inf _{t} \lambda_{\min }\left(B^{\top}\left(A_{m}+B \bar{K}_{x}\right)^{-\top} \mathscr{Q}\left(A_{m}+B \bar{K}_{x}\right)^{-1} B\right)>$ $0, \quad$ and $\quad c_{3}=\frac{\sup _{t}\left\|B^{\top} W\left(A_{m}+B \bar{K}_{x}\right)^{-1} B\right\|\left\|\Theta^{*}\right\|}{c_{2}}>0$. Then, $\|\Phi(x)\| \leq \Phi_{0}$ for $0<v<v_{\max }$. Thus, $\dot{V} \leq 0$ outside a compact set. Therefore, the closed-loop system with the optimal control modification is uniformly ultimately bounded with the following ultimate bounds:

$$
\begin{gathered}
\|e\| \leq \sqrt{\frac{\lambda_{\max }\left(W^{*}\right) p^{2}+\lambda_{\max }\left(\Gamma^{-1}\right) \alpha^{2}}{\lambda_{\min }\left(W^{*}\right)}} \\
\|\tilde{\Theta}\| \leq \sqrt{\frac{\lambda_{\max }\left(W^{*}\right) p^{2}+\lambda_{\max }\left(\Gamma^{-1}\right) \alpha^{2}}{\lambda_{\min }\left(\Gamma^{-1}\right)}}
\end{gathered}
$$

where $p=\sqrt{\frac{v c_{2} c_{3}^{2} \Phi_{0}^{2}}{c_{1}}}$ and $\alpha=2 c_{3}$. When $v=0$, we recover the new MRAC update law. Then, it can be shown that $\ddot{V}$ is bounded. Invoking the Barbalat's lemma, $\dot{V} \rightarrow 0$ which implies $e \rightarrow 0$ as $t \rightarrow \infty$. Thus, asymptotic tracking is achieved with the time-varying reference model modification by the performance optimizing controller for the new MRAC update law.

\section{APPLICATION}

A maneuver load alleviation adaptive control design is implemented for a flexible wing Generic Transport Model (GTM) equipped with the Variable Camber Continuous Trailing Edge Flap (VCCTEF) [6] as shown in
Fig. 1. Maneuver load alleviation can be viewed as a performance optimizing control objective to maintain structural load limits on aircraft wings during a maneuver. A pull-up maneuver is simulated with the VCCTEF deployed for the maneuver load alleviation while the elevator is deployed for the pitch rate control.

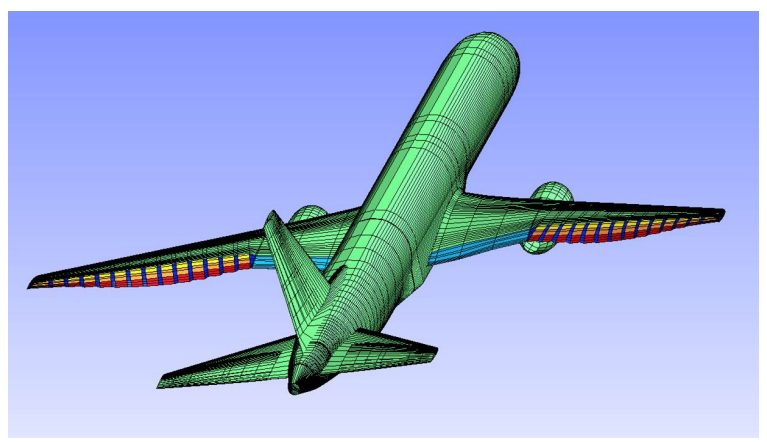

Figure 1. GTM with Variable Camber Continuous Trailing Edge Flap

The longitudinal aircraft model is expressed as

$$
\dot{x}=A x+B_{p} u_{p}+B_{a}\left(u_{a}+\Theta^{* \top} x\right)
$$

where $x=\left[\begin{array}{lllll}h & V & \alpha & q & \theta\end{array}\right]^{\top}$ is the aircraft state vector with $h$ as the altitude, $V$ the airspeed, $\alpha$ the angle of attack, $q$ the pitch rate, $\theta$ the pitch attitude; $u_{a}=\delta_{e}$ is the elevator deflection; $u_{p}$ is the shape function of the VCCTEF deflections, and $\Theta^{*}=$ $\left[\begin{array}{lllll}0 & 0 & \theta_{\alpha}^{*} & \theta_{q}^{*} & 0\end{array}\right]^{\top}$ is a matched uncertainty.

A pitch attitude controller is designed for the elevator to track a second-order pitch attitude reference model

$$
\ddot{\theta}_{m}+2 \zeta \omega_{n} \dot{\theta}_{m}+\omega_{n}^{2} \theta_{m}=\omega_{n}^{2} r
$$

where $\zeta$ is the desired closed-loop damping ratio and $\omega_{n}$ is the desired closed-loop frequency. The adaptive controller for the elevator is designed as

$$
u_{a}=K_{x} x+k_{r} r-\Theta^{\top} x
$$

For the maneuver load alleviation, the performance metric is the wing root bending moment which can be measured from a strain gauge sensor. Then, the performance optimizing controller is designed as

$$
u_{p}=\bar{K}_{x} x+\bar{K}_{z} z+\bar{K}_{z_{r}} z_{r}
$$

where the reference command generating function is specified as

$$
\left[\begin{array}{c}
\dot{z} \\
\dot{r}
\end{array}\right]=\left[\begin{array}{cc}
0 & \omega \\
-\omega & 0
\end{array}\right]\left[\begin{array}{l}
z \\
r
\end{array}\right]+\left[\begin{array}{c}
0 \\
\theta_{0} \omega
\end{array}\right]
$$

with $\theta_{0}=20^{\circ}$ and $\omega=2 \mathrm{rad} / \mathrm{sec}$. The weighting coefficient and matrix are selected to be $Q=6 \times 10^{-12}$ and $R=1000 I . \Theta, \hat{C}$, and $\hat{D}$ are initialized with zero values. The adaptation rate matrices are chosen to be $\Gamma=$ 
$\operatorname{diag}(0,0,0.2,0.5,0), \quad \Gamma_{C}=\operatorname{diag}(10,10000,2000,5,1)$, and $\Gamma_{D}=\operatorname{diag}(30,40,30,50)$. The optimal control modification adaptive law is implemented with $v=0.01$.

Figure 2 shows the time histories of the aircraft pitch attitude and the wing root bending moment. The aircraft pitch attitude tracks the time-varying modified reference model very well with the performance optimizing adaptive controller. The amplitude of the wing root bending moment is effectively reduced by $38.8 \%$ due to the performance optimizing adaptive controller. Thus, the effectiveness of the performance optimizing adaptive controller is demonstrated. The estimated performance metric $\hat{y}$ agrees very well with the measured performance metric $y$. Figure 3 shows the time histories of the adaptive parameters $\Theta, \hat{C}$, and $\hat{D}$ which converge to their steady state values. Figure 4 shows the control signals of the elevator deflection $\delta_{e}$ and the VCCTEF deflections $\delta$. All the control signals appear to be well-behaved.
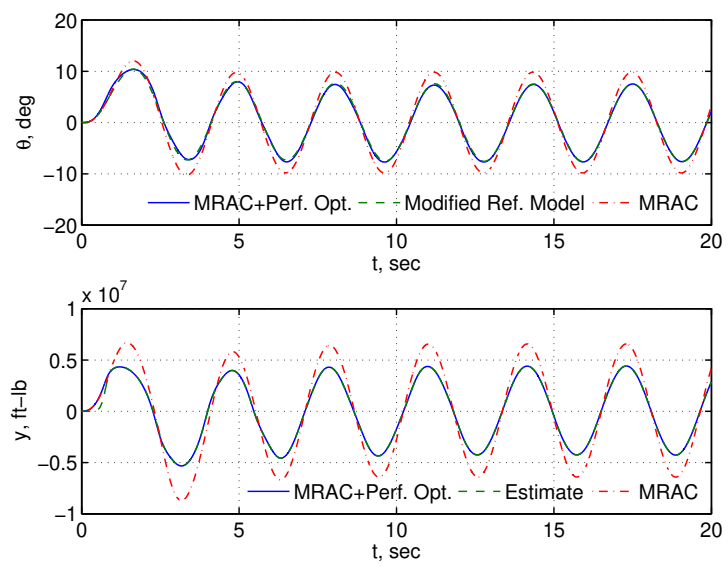

Figure 2. Aircraft Response to Performance Optimizing Adaptive Control with $r(t)=\theta_{0} \sin \omega t$
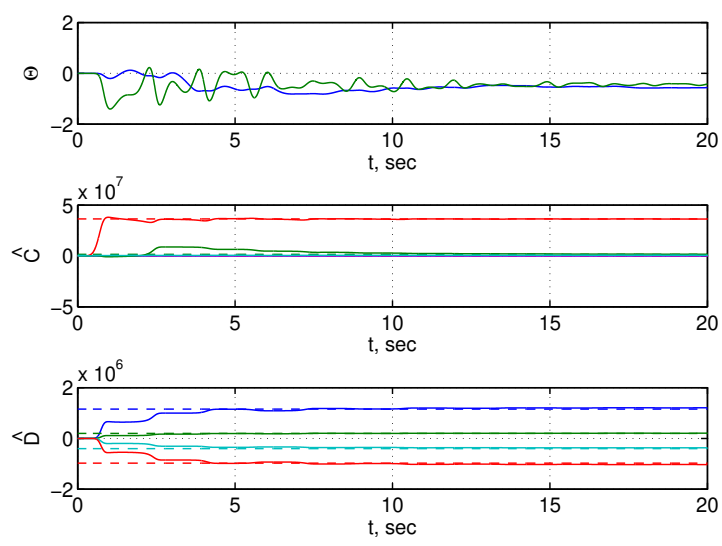

Figure 3. $\Theta(t), \hat{C}(t)$, and $\hat{D}(t)$ with $r(t)=\theta_{0} \sin \omega t$
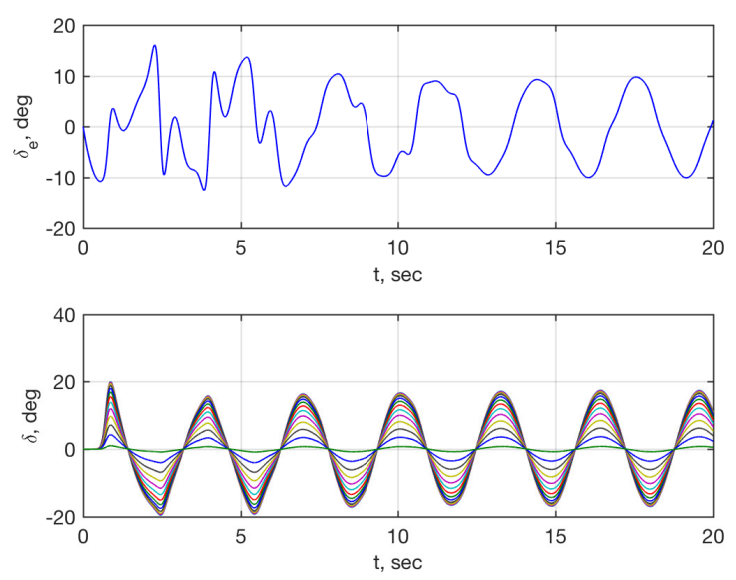

Figure 4. $\Theta(t), \hat{C}(t)$, and $\hat{D}(t)$ with $r(t)=\theta_{0} \sin \omega t$

\section{Conclusions}

This paper presents a new adaptive control method that includes a performance optimization objective. The performance optimization is formulated as a multi-objective optimal control problem coupled with a least-squares parameter estimation of the unknown sensitivities of the performance metric. The gradient optimization leads to a set of modified time-varying Riccati and Sylvester equations for which the standard weighting matrices $Q$ and $R$ are modified to incorporate the estimates of the performance metric sensitivities. The resultant timevarying modification of the reference model necessitates a revision of the standard MRAC using a time-varying weighting matrix computed from the modified Riccati equation. The revised MRAC achieves simultaneously asymptotic tracking and performance optimization. The effectiveness of the proposed method is demonstrated in simulations of maneuver load alleviation control for a flexible wing Generic Transport Model.

\section{REFERENCES}

[1] Lavretsky, E. and Wise, K., Robust and Adaptive Control, Springer, 2012.

[2] Johnson, E. N. and Calise, A. J., "Pseudo-Control Hedging: A New Method for Adaptive Control," Advances in Navigation Guidance and Control Technology Workshop, November 2000.

[3] Nguyen, N. and Balakrishnan, S. N.,'Bi-Objective Optimal Control Modification Adaptive Control for Systems with Input Uncertainty," IEEE/CAA Journal of Automatica Sinica, Vol. 1, No. 4, pp. 423-434, October 2014.

[4] Lewis, F. L., Vrabie, D. L., and Syrmos, V. L., Optimal Control, Third Edition, John Wiley \& Sons, Inc., 2012

[5] Nguyen, N., "Optimal Control Modification for Robust Adaptive Control with Large Adaptive Gain," Systems \& Control Letters, 61 (2012) pp. 485-494.

[6] Nguyen, N., "Elastically Shaped Future Air Vehicle Concept," NASA Innovation Fund Award 2010 Report, October 2010, Submitted to NASA Innovative Partnerships Program, http://ntrs.nasa.gov/archive/nasa/casi.ntrs.nasa.gov/20110023698.pdf 